\title{
HAPTIC GUIDANCE BASED ON HARMONIC FUNCTIONS FOR THE EXECUTION OF TELEOPERATED ASSEMBLY TASKS
}

\author{
Carlos Vázquez* Jan Rosell ${ }^{*, 1}$ \\ * Institute of Industrial and Control Engineering \\ Technical University of Catalonia (IOC-UPC) \\ Barcelona, SPAIN, Email: jan.rosell@upc.edu
}

\begin{abstract}
Haptic devices allow the user to feel the reaction forces and torques that arise when the virtual object attached to the user-manipulated probe touches the other objects in the virtual environment. Additionally, the user may feel some guiding constraints and forces that aid him in the completion of a virtual task. Also, haptic devices are used as master devices in teleoperation tasks and may include force reflection from the real forces sensed by a force sensor located at the robot wrist. Both operation modes can be combined to set up an assisted teleoperation system able to execute assembly tasks. This paper proposes the use of path planning techniques based on harmonic functions to generate a guiding force that aids the user during the teleoperation.
\end{abstract}

Keywords: Haptic devices, teleoperation, assembly tasks, path planning, harmonic functions.

\section{INTRODUCTION}

During the last decade robotics has extended its potential applications by the increasing use of haptic devices, either in medicine, industry, entertainment or education (see (Srinivasan and Basdogan, 1997) for a taxonomy and first introduction to the field of haptics). Haptic devices are being used both as master devices for the teleoperation of robotic manipulators, or in the execution of virtual tasks. Haptic devices provide the user with force feedback, allowing him to feel the reaction forces that arise when the teleoperated device interacts with the environment (e.g. (Elhajj et al., 2001)(Font et al., 2004)), or to feel those artificial forces that should be sensed when the virtual manipulated object interacts with the virtual en-

\footnotetext{
1 This work was partially supported by the CICYT projects DPI2004-03104 and DPI2005-00112.
}

vironment (e.g. (Ruspini et al., 1997)(Otaduy and Lin, 2006)).

Besides these reaction forces, either from the real or virtual world, the haptic device can also exert some forces to aid the user in the execution of the teleoperated task. Some simple guiding forces may constrain the user motions along a line or curve or over a given working plane or surface, e.g. for a peg-in-hole task a line can be defined along the axis of the hole and the user may feel an increasing force as he moves the robot tool with the peg away from that line. Although these simple guides can already be a good help, some teleoperated tasks may require more demanding guiding forces to aid the user all along the task execution. This is, for instance, the case with assembly tasks where the part being manipulated by the robot must either perform compliant motions to successfully accomplish the 


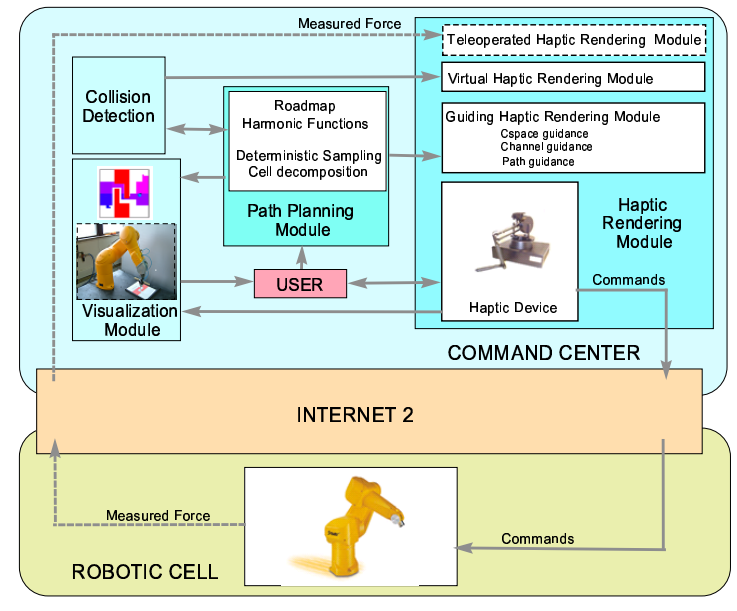

Fig. 1. System setup.

task (Galeano and Payandeh, 2005), or move on a micro or nano-scale (Varol et al., 2006).

With this assistance aim, this paper proposes the generation of a guiding force using path planning techniques based on harmonic functions. This harmonic functions are computed over an approximate cell decomposition of Configuration Space $(\mathcal{C}$-space $)$. A simple method is then introduced to generate the guiding force from the harmonic function values of the cells of the $\mathcal{C}$-space decomposition.

\section{APPROACH OVERVIEW}

Fig. 1 shows the assisted teleoperation system setup, with the two main modules that currently constitute it:

Path Planning Module. This module receives as input the model of the assembly task to be teleoperated and its starting and goal configurations. It outputs a path that connect those configurations, a channel of cells that connect the cells that contain them, and two harmonic functions.

Haptic Rendering Module. This module sends to the haptic device the combination of the following forces: a) the real force sensed by the force sensor attached to the wrist of the robot; b) a guiding force computed from the output of the path planning module; and c) a virtual reaction force computed from the interpenetration between the manipulated object and the obstacles in the virtual environment.

The user reacts at that force moving the haptic device accordingly. The motions done are sent to the teleoperated robot. Although this is the intended complete system, currently the teleoperation is not yet bilateral, i.e. the real forces measured by the sensor attached at the robot wrist are not fed back (indicated with dashed lines in Fig. 1).
The teleoperation scheme set up at the IOC's Robotic Lab is described in (Nuño and Basañez, 2006). It makes use of a communication channel passivation done using wave variables with an impedance adaptation and TCP/UDP and IPv6 protocols.

The paper describes the main ideas of the path planning module in Section 3 and presents the haptic rendering module in Section 4. Finally, Section 5 illustrates the approach in a simple 2D example and Section 6 concludes the work.

\section{PATH PLANNING}

The planning of collision-free paths for a manipulated part through the other parts or obstacles in an assembly is a difficult problem that is usually tackled using a configuration space approach. In $\mathcal{C}$-space the manipulated part is mapped to a point and the obstacles are enlarged accordingly $(\mathcal{C}$-obstacles $)$. The $\mathcal{C}$-space explicitly captures the motion freedom of the manipulated part, which facilitates path planning.

Of the several path planning techniques that exist, those based on potential field methods are the best alternatives, although not for very high dimensional $\mathcal{C}$-spaces since usually they require an approximate decomposition of $\mathcal{C}$-space. Among them, those based on harmonic functions are interesting because they give rise to practical, resolution-complete planners without local minima (Connolly et al., 1990). To compute the $\mathcal{C}$-space decomposition, hierarchical structures can be considered in order to reduce the number of cells; also, the combination with sampling techniques may overcome the requirement of the difficult characterization of the $\mathcal{C}$-obstacles (Rosell and Iñiguez, 2005). The solution found by these planners is a channel of cells connecting the cell that contains the initial configuration with the cell that contains the goal configuration.

Another family of successful path planners are those based on the sampling of $\mathcal{C}$-space and the construction of a roadmap (Kavraki et al., 1996). The roadmap is obtained by connecting the free sampled configurations with free paths using a simple local planner. The initial and the goal configurations are then connected to the roadmap and a free path connecting them can be found using graph searching techniques.

The path planning module considered in this paper, described in detail in (Rosell et al., 2007a), combines a sampling based approach with an harmonic function based approach. The combination relies on the alternating execution of two steps:

a) The exploration of the $\mathcal{C}$-space and the construction of the hierarchical cell decomposition 
that consists in the iterative sampling of configurations, their classification into cells and in the partition of those cells that are not homogeneous enough (Section 3.1).

b) The computation of an harmonic function to find a channel connecting the cell containing the initial configuration with the cell containing the goal one (Section 3.2).

\subsection{Cell decomposition}

The $\mathcal{C}$-space is considered as a $d$-dimensional unit cube; it is partitioned using a $2^{d}$-tree decomposition. The initial cell with sides with unitary size is the tree root. The levels in the tree are called partition levels and are enumerated such that the tree root is the partition level 0 and the maximum resolution ${ }^{2}$ corresponds to partition level $M$. Cells of a given partition level $m$ are called $m$-cells.

The $2^{d}$-tree decomposition is obtained by the iterative sampling of configurations and partitioning of cells. Sampling is performed using a deterministic sequence that uniformly and gradually covers the $\mathcal{C}$-space (Rosell et al., 2007b).

Partitioning is done as follows. The proposed approach does not classify cells between free and obstacle cells, but with a continuous parameter, called transparency, computed as a function of the number of free or obstacle samples that the cell contains, i.e. it captures the homogeneity of a cell:

$$
T_{j}=\frac{\sum_{i=1}^{i=K_{j}} \text { color }_{i}}{K_{j}}
$$

with $K_{j}$ being the number of samples of the cell and

color $_{i}=\left\{\begin{array}{l}+1 \text { if } c_{i} \text { is a free configuration } \\ -1 \text { if } c_{i} \text { is an obstacle configuration }\end{array}\right.$

When the transparency is within a given interval around zero the cell is considered to be not homogeneous enough and then it is partitioned into its $2^{d}$ descendant cells, and the transparency for each descendant cell is then recomputed.

\subsection{Harmonic functions}

An harmonic function $\phi$ on a domain $\Omega \subset \mathrm{R}^{n}$ is a function that satisfies Laplace's equation:

2 The maximum resolution needed is a fixed value determined by the clearance of the path planning problem to be solved.

$$
\nabla^{2} \phi=\sum_{i=1}^{n} \frac{\partial^{2} \phi}{\partial x_{i}^{2}}=0
$$

The solution of the Laplace's equation is usually found numerically using finite difference methods, i.e. by constraining $\phi$ on a regular grid and using relaxation methods that iteratively update the value of a cell as the mean of its neighbor cells. An extension to non-regular grids is also possible, as done in (Rosell and Iñiguez, 2005) using a $2^{d}$-tree decomposition.

In this paper the same approach is followed, although the harmonic function is not only computed over the free cells (fixing the obstacle cells at a high value), as it is usually done, since this cell classification is not considered here. Instead, the harmonic function values (called HF-values) are computed over the whole set of cells using the transparency as a weighting parameter, except for the goal cell that is fixed at a low value $U_{L}=-1$. The computation is done as follows:

First, for any cell $C_{j}^{m}$ a weighted mean, $U_{j}$, of the HF-values of the cells neighboring $C_{j}^{m}$ is computed. The sizes of the border between cells are used as weights, such that the bigger a neighbor cell is the greater influence it has on $U_{j}$ :

$$
U_{j}=\frac{\sum_{i=1}^{N_{j}} \omega_{i, j} u_{i, j}}{\sum_{i=1}^{N_{j}} \omega_{i, j}}
$$

with $N_{j}$ being the number of neighbors, $u_{i, j}$ the harmonic function value of a neighbor cell $C_{i}^{n}$, and $\omega_{i, j}$ the size of the border between $C_{i}^{n}$ and $C_{j}^{m}$ measured in $M$-cells:

$$
\omega_{i, j}=2^{(d-1)(M-\max (m, n))}
$$

Then, the HF-value of the cell is computed in such a way that the more transparent the cell is the more it is influenced by its neighbors and the less it is fixed at a high potential value, i.e. if $U_{H}$ is the high value of the harmonic function (fixed at $\left.U_{H}=0\right)$, the HF-value is computed as:

$$
H_{j}=\frac{1}{2}\left[U_{j}\left(1+T_{j}\right)+\left(1-T_{j}\right) U_{H}\right]
$$

with $T_{j} \in[-1,1]$ being the transparency of $C_{j}^{m}$.

Using this procedure, two harmonic functions are computed over the $\mathcal{C}$-space:

HF1: This harmonic function is computed by considering as goal cell the cell that contains the goal configuration, i.e. the HF-value of this cell is set at the low value $U_{L}$. The HF-values of all the other cells are computed by relaxation, i.e. by iteratively (re)computing them until a channel (called solution channel) from the cell containing the initial configuration to the goal cell is found. 
The solution channel is computed following the negated gradient of the harmonic function, i.e. starting at the initial cell each next cell in the channel is selected as the neighboring one with lowest $\mathrm{HF}$-value.

HF2: This harmonic function is computed by considering as goal cells all the cells of the channel obtained using HF1. i.e. the HF-value of these cells are set at the low value $U_{L}$. The HF-values of all the other cells are also computed by relaxation.

Following the negated gradient of HF2 from any cell leads to the nearest cell of the solution channel.

\subsection{Roadmap}

The samples contained inside the channel cells are connected using a simple local planner. Since the transparency of channels cells is high, these samples are usually connected successfully. The resulting roadmap is searched using the $\mathrm{A}^{*}$ algorithm to obtain a solution path connecting the initial and the goal configurations.

\section{THE HAPTIC GUIDANCE}

It is assumed that the user allways starts the assembly task around a given starting configuration, and that the task completion is determined by a given goal configuration. Those configurations and the task model are the input to the path planning module, being the outputs: a) the two harmonic functions HF1 and HF2; b) the channel of cells that connects the cell containing the initial configuration with the cell that contains the goal configuration; and c) a solution path through the channel that connects the starting and goal configurations and that is computed using the configurations of the channel cells.

This path planning output is used by the haptic module to compute the haptic guidance, that is performed at three levels:

$\mathcal{C}$-space guidance: It is felt on all the cells of $\mathcal{C}$-space that do not pertain to the solution channel. This guiding force pushes the user towards the solution channel. It is computed using $\mathrm{HF} 2$.

Channel guidance: It is felt on the cells of the channel. This force guides the user within the channel towards the goal cell. It is computed using HF1.

Path guidance: It is felt near the path and pushes the user towards it.

The two first guidances are done using the cell decomposition and the harmonic functions (HF2
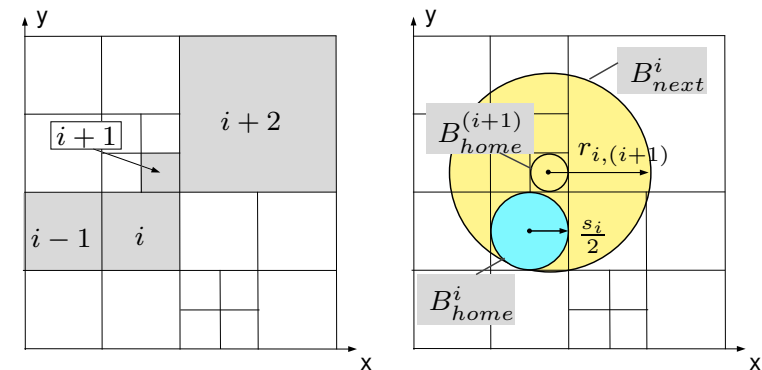

Fig. 2. Solution channel (left); balls $B_{\text {home }}^{i}$ and $B_{\text {next }}^{i}$ (right).

for the $\mathcal{C}$-space guidance and HF1 for the channel guidance). The procedure is detailed in Section 4.1. The last one is done using the solution path and is explained in Section 4.2. Both type of guiding procedures are implemented using simple haptic programming primitives:

Point attraction primitive: generates a force directed towards a given configuration when the haptic interface point is within a given distance from it.

Segment attraction primitive: generates a force directed towards a given segment defined by its two extreme configurations, provided that the haptic interface point is within a given distance from the segment.

\subsection{Cell guidance}

The cell guidance iteratively uses the point attraction primitive to generate attractive force towards the centers of the cells following the negated gradient of the harmonic functions.

The procedure is the following. For each cell $i$ with side $s_{i}$ the following two balls are defined:

- $B_{\text {home }}^{i}$ : A ball of radius $\frac{s_{i}}{2}$ centered at the cell center.

- $B_{n e x t}^{i}$ : A ball centered at the center of the neighbor cell $j$ with lowest HF-value and radius $r_{i,(i+1)}=d_{i,(i+1)}+\frac{s_{i}}{2}$, with $d_{i,(i+1)}$ being the distance between centers ${ }^{3}$ (Figure 2).

Then, starting within the ball $B_{h o m e}^{i}$ of a given cell $i$, an attractive force is set that guides the haptic device towards the center of $B_{n e x t}^{i}$. This force is felt inside $B_{n e x t}^{i}$ until the haptic interface point enters the ball $B_{\text {home }}^{(i+1)}$ (note that $B_{\text {next }}^{i}$ and $B_{\text {home }}^{(i+1)}$ are concentric balls). At this point the force changes to become an attractive force centered at $B_{\text {next }}^{(i+1)}$.

\footnotetext{
3 Note that by construction the ball $B_{n e x t}^{i}$ always contains $B_{\text {home }}^{i}$, which guarantees the continuity in the guiding procedure.
} 


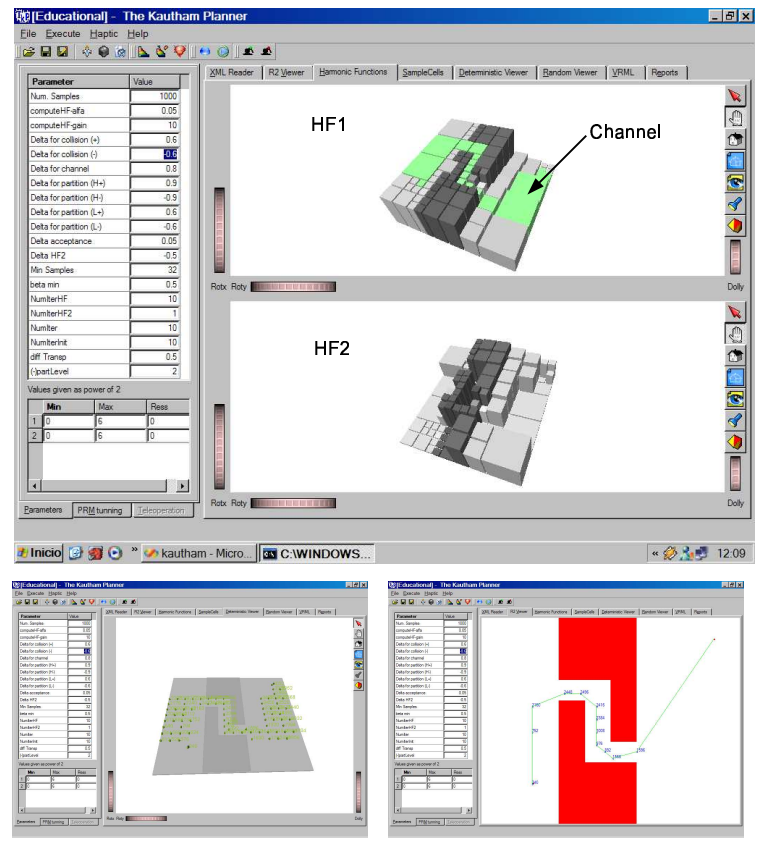

Fig. 3. Top: Harmonic functions HF1 and HF2; Bottom: samples on the solution channel and the path obtained.

This procedure is repeated iteratively until a final cell is reached (for the $\mathcal{C}$-space guidance the final cells are the channel cells; for the channel guidance the final cell is the goal cell). Final cells have empty $B_{\text {next }}$ balls.

\subsection{Path guidance}

The solution path output by the path planning module is a set of linear segments of $\mathcal{C}$-space. The path guidance uses a simple attractive force towards that set of segments using the segment attraction primitive.

\section{VALIDATION}

The software has been implemented in $\mathrm{C}++$ using the cross-platform tools Qt (as application framework), Coin3D (as graphics toolkit) and OpenHaptics (Itkowitz et al., 2005) as haptics library.

As an example a 2D bend corridor has been considered. The task has been defined directly over $\mathcal{C}$-space, i.e. the manipulated object is a point that has to move from right to left through the corridor. This greatly simplifies the collision detection tests and the computation of the virtual reaction forces when the manipulated point tries to interpenetrate the obstacles defining the corridor. Thus, the example focuses on the guiding part of the haptic rendering module.

Figure 3 (top) shows the harmonic functions HF1 and HF2. The solution channel is highlighted in green over HF1. As it can be observed on HF2,
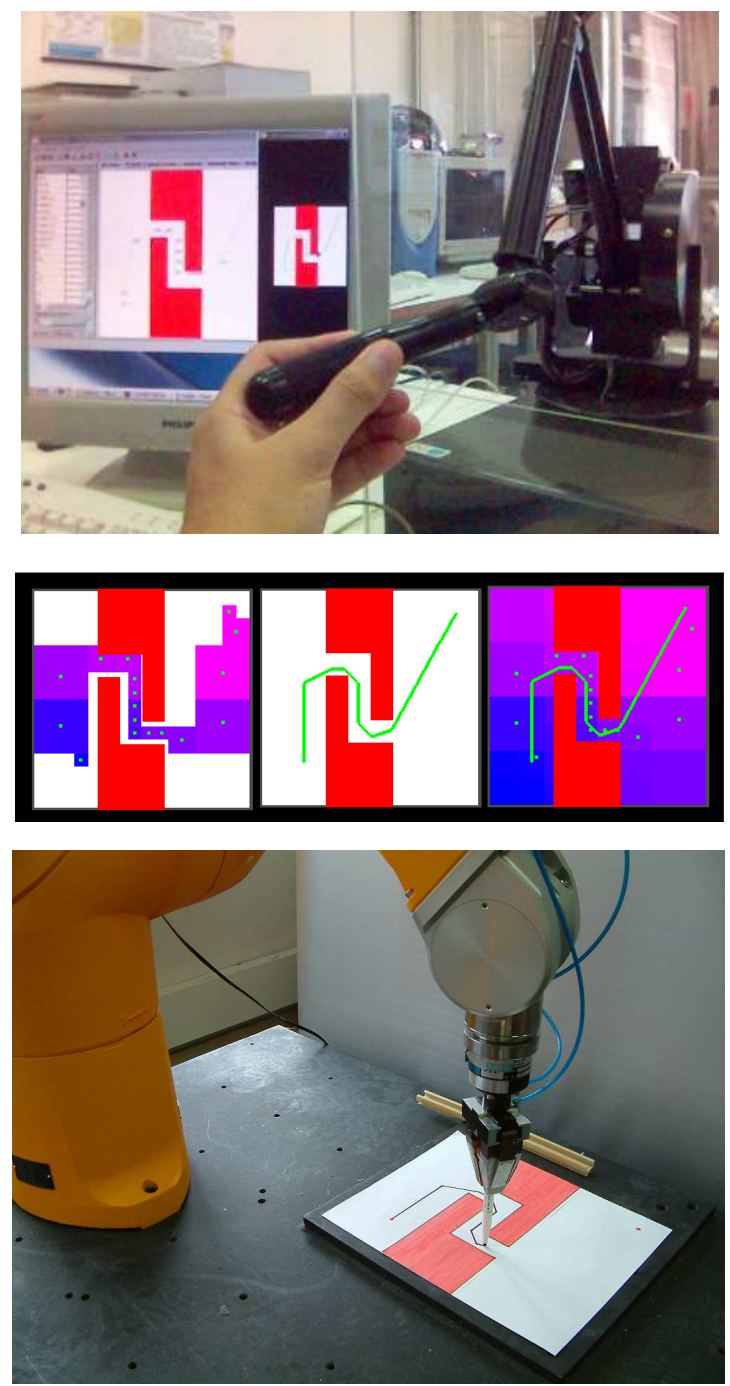

Fig. 4. Channel and path guidance for the teleoperation using the Phantom haptic device teleoperating a Stäubli TX-90 robot.

the channel cells have a low HF2-value. Figure 3 (bottom) shows the samples over the solution channel and the solution path found using them.

Figure 4 shows the user manipulating the Phantom haptic device (top) and the Stäubli TX-90 robot executing the task (bottom). The haptic guidances are shown between the two photographs (the channel guidance on the left, the path guidance on the middle and the three together on the right).

Extensions to 6 d.o.f. tasks are currently being implemented. The path planning module is already applicable as is (and therefore the guiding haptic rendering too). The virtual part of the haptic rendering module is being modified to compute the virtual reaction forces that appear when the manipulated part interacts with the obstacles in the virtual environment. The procedure followed is based on the use of configuration space (Rosell and Vázquez, 2005). 


\section{CONCLUSIONS}

The combined use of a haptic device both as a teleoperation master device and as a way to interact with virtual worlds allows the set up of an assisted teleoperation system. This paper has introduced the use of a path planning technique based on harmonic functions to generate guiding forces that aid a user in the teleoperation of assembly tasks. Two harmonic functions are computed over a hierarchical cell decomposition of $\mathcal{C}$-space: a) one to find a solution channel (sequence of cells) that connects the cell containing the initial configuration to the cell containing the goal configuration; b) the other has all the cells of the solution channel as goal cells and is aimed at guiding the motions towards the channel. Also, a path is computed within the channel connecting the initial and the goal configurations.

From these harmonic functions and from the solution path, haptic guidance is performed at three levels: a) as a force felt on all the cells of $\mathcal{C}$-space that pushes the user towards the solution channel; b) as a force felt on the cells of the channel guiding the user within the channel towards the goal cell; c) a force felt near the path guiding the user towards it.

The approach has been validated with a 2D bendcorridor problem, using a Phantom haptic device. The motions of the haptic device exerted by the user (and directed by the guiding forces) are sent to a Stäubli TX-90 robot using a teleoperation scheme over the internet using IPv6 protocols.

\section{REFERENCES}

Connolly, C. I., J. B. Burns and R. Weiss (1990). Path planning using Laplace's equation. In: Proc. of the IEEE Int. Conf. on Robotics \& Automation. pp. 2102-2106.

Elhajj, I., N. Xi, W. K. Fung, Y. H. Liu, W.J. Li, T. Kaga and T. Fukuda (2001). Haptic information in internet-based teleoperation. IEEE/ASME Transactions on Mechatronics 6(3), $295-304$.

Font, I., S. Weiland, M. Franken, M. Steinbuch and L. Rovers (2004). Haptic feedback designs in teleoperation systems for minimal invasive surgery. In: IEEE Int. Conf. on Systems, Man and Cybernetics. Vol. 3. pp. 2513 -2518 .

Galeano, D. and S. Payandeh (2005). Artificial and natural force constraints in haptic-aided path planning. In: IEEE Int. Workshop on
Haptic Audio Visual Environments and their Applications.

Itkowitz, B., J. Handley and W. Zhu (2005). The OpenHaptics toolkit: a library for adding 3D touch navigation and haptics to graphics applications. In: Proc. of the First Joint Eurohaptics Conference and Symposium on Haptic Interfaces for Virtual Environment and Teleoperator Systems. pp. $590-591$.

Kavraki, L. E., P. Svestka, J.-C. Latombe and M. K. Overmars (1996). Probabilistic roadmaps for path planning in high - dimensional configuration spaces. IEEE Trans. on Robotics and Automation 12(4), 566-580.

Nuño, E. and L. Basañez (2006). Force feedback to assist teleoperation systems via high speed networks. In: Proc. of the IFR/DGR 37th Int. Symp. on Robotics.

Otaduy, M.A. and M. C. Lin (2006). A modular haptic rendering algorithm for stable and transparent 6-dof manipulation. IEEE Transactions on Robotics 22(4), 751 - 762.

Rosell, J. and I. Vázquez (2005). Haptic rendering of compliant motions using contact tracking in Cspace. In: Proc. of the the IEEE Int. Conf. on Robotics and Automation. pp. 42234228.

Rosell, J. and P. Iñiguez (2005). Path planning using harmonic functions and probabilistic cell decomposition. In: Proc. of the IEEE Int. Conf. on Robotics and Automation. pp. 18151820.

Rosell, J., C. Vázquez, A. Pérez and P. Iñiguez (2007a). A novel path planning proposal based on the combination of deterministic sampling and harmonic functions. Technical report. IOC-DT-P-2007-06.

Rosell, J., M. Roa, A. Pérez and F. García $(2007 b)$. Sdk: A proposal of a general and efficient deterministic sampling sequence. Technical report. IOC-DT-P-2007-07.

Ruspini, D. C., K. Kolarov and O. Khatib (1997). The haptic display of complex graphical environments. In: Proc. of the 24th annual Conf. on Computer graphics and Interactive Techniques. pp. 345-352.

Srinivasan, M. A. and C. Basdogan (1997). Haptics in virtual environments: Taxonomy, research status, and challenges. Computer and Graphics 21(4), 393-404.

Varol, A., I. Gunev and C. Basdogan (2006). A virtual reality toolkit for path planning and manipulation at nano-scale. In: 14th Symp. on Haptic Interfaces for Virtual Environment and Teleoperator Systems. pp. $485-489$. 